\title{
Treatment of acne with intermittent and conventional isotretinoin: a randomized, controlled multicenter study
}

\author{
Ayse Akman · Cicek Durusoy • Meltem Senturk • \\ Cilem Kaya Koc $\cdot$ Durali Soyturk $\cdot$ Erkan Alpsoy
}

Received: 9 May 2007 / Revised: 3 July 2007 / Accepted: 30 July 2007 / Published online: 21 August 2007

(C) Springer-Verlag 2007

\begin{abstract}
Oral isotretinoin is the most effective choice in the treatment of severe acne. Application of isotretionin to acne has been expanded to treat those patients with less severe but scarring acne who are responding unsatisfactorily to conventional therapies. However, its use is associated with many side effects, some of which can result in very disastrous consequences. Data related with intermittent isotretinoin therapy is still limited. Our aim was to asses the efficacy and tolerability of two different intermittent isotretinoin courses and compare them with conventional isotretinoin treatment. In this multicenter and controlled study, 66 patients with moderate to severe cases were randomized to receive either isotretionin for the first 10 days of each month for 6 months (group 1), or each day in the first month, afterwards the first 10 days of each month for 5 months (group 2) or daily for 6 months (group 3). The drug dosage was $0.5 \mathrm{mg} / \mathrm{kg} / \mathrm{day}$ in all groups. Patients were followed-up for 12 months. Efficacy values were evaluable for 22 patients in group 1, 19 patients in
\end{abstract}

The study was supported by Akdeniz University Scientific Research Projects Unit.

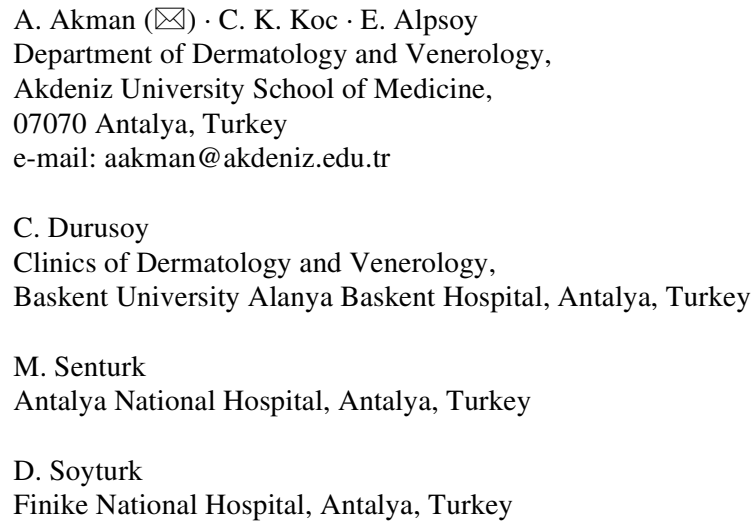

group 2, and 19 patients in group 3. Acne scores in each group were significantly lower at the end of treatment and follow-up periods $(P<0.001)$. When patients were evaluated separately as moderate $(n=31)$ and severe $(n=29)$, no statistically significant differences were obtained among the treatment protocols in patients with moderate acne. However, there was a significant difference between groups 1 and 3 to the response of the treatments in severe acne patients at the end of follow-up period $(P=0.013)$. The frequency and severity of isotretionin-related side effects were found to be lower in groups 1 and 2 compared with group 3 . Intermittent isotretinoin may represent an effective alternative treatment, especially in moderate acne with a low incidence and severity of side effects. The intermittent isotretinoin can be recommended in those patients not tolerating the classical dosage.

\section{Introduction}

Oral isotretinoin is the most effective choice in the treatment of severe acne [7]. Acne especially in more severe forms has the potential to result in scarring and permanent disfigurement. Previous studies showed that combination therapies are a highly effective treatment in severe forms of acne. However, scarring can occur in patients with acne who had not responded to conventional antibiotic therapy and combination treatments [4]. This condition can cause significant embarrassment and anxiety when the affected population is considered. Therefore, effective treatment is necessary to prevent significant psychological and social impairment in these patients. Application of the drug to acne has been expanded to treat those patients with less severe but scarring acne who are responding unsatisfactorily to conventional therapies $[2,5]$. However, its use is associated with many 
side effects, some of which can result in very disastrous consequences. Because of the side effects and the necessity of long-term daily use of this drug, patients can have difficulties in complying with the treatment.

Recent reports indicate that acne patients have been benefiting from the low dose or intermittent treatment protocols. Low dose isotretinoin, such as $0.15-0.40 \mathrm{mg} / \mathrm{kg}$ was reported to be effective with a low incidence of severe side effects [2, $7,10]$. Strauss et al. [11] showed that single daily doses of $0.4 \mathrm{mg} / \mathrm{kg}$ of micronized isotretinoin is effective in patients with severe recalcitrant nodular acne with a lower risk of mucocutaneous events, and hypertriglyceridemia, than in those receiving standard isotretinoin. Data related with intermittent isotretinoin therapy is still limited. Goulden et al. [5] and recently Kaymak et al. [6] in their open studies showed that intermittent protocols are effective in mild and moderate acne with mild side-effects. They administered isotretinoin for 1 week in every 4 weeks for a total period of 6-month. Goulden et al. [5] used the drug at a dosage of $0.5 \mathrm{mg} / \mathrm{kg} / \mathrm{day}$ and Kaymak et al. [6] at $0.5-0.75 \mathrm{mg} / \mathrm{kg} / \mathrm{day}$. These studies, however, were not planned as controlled trials, and included only mild to moderate acne cases. On the other hand, the study of Kaymak et al. did not follow up the patients.

In the present multicenter, randomized and controlled study, we aimed to asses the efficacy and tolerability of two different intermittent isotretinoin courses and compare them with conventional isotretinoin treatment. Besides moderate cases, we also included severe cases in the study, and all patients were followed-up for a period of 12-months.

\section{Patients and methods}

This was a multicenter, randomized and controlled study consisting of 66 patients with the FDA global grade 2 (moderate) and 3-4 (severe) [3]. Patients with acne who had not responded to conventional antibiotic therapy or who had rapidly relapsed after conventional treatment were included in the study. Patients were excluded if they had acne conglobata, acne fulminans or systemic disorders requiring any treatment. An appropriate washout period before the treatment for patients who were receiving any conventional treatments was applied before treatment began. Patients in group 1 were treated for the first 10 days of each month for 6 months. Group 2 were given isotretinoin each day in the first month, afterwards the first 10 days of each month for 5 months. In group 3, a conventional isotretinoin treatment course was used for 6 months. Isotretinoin was administered at a dose of $0.5 \mathrm{mg} / \mathrm{kg} /$ day in all groups (Fig. 1). Standard moisturizing and washing procedures were recommended for each patient.

Facial lesions were taken into consideration. To evaluate the FDA global grade, inflammatory (papules, pustules, nodules), and non-inflammatory lesions (comedones) were counted at each clinic visit by the same investigator in each clinic. The FDA global grade was performed as previously described [3]. The patients were examined clinically for acne grading at the baseline, afterwards, every month during therapy, and every 3 months for a 12-month follow-up period.

During treatment, physicians recorded side effects at weeks 2, 4, 8, 12, 16, 20 and 24. Each side effect was evaluated on a 4-point scale as none (0), mild (1), moderate (2) or severe (3). Side effects were also scored by the patients using a $10-\mathrm{cm}$ visual anologue scale (VAS), ranging from none to extremely noticable on a weekly basis during the treatment.

Liver function tests and fasting lipids were evaluated at the baseline, and afterwards, monthly during the treatment. A pregnancy test was performed at the beginning, contra-
Fig. 1 Flow-chart of patients participating in the study. $\mathrm{m} / \mathrm{s}$ moderate/severe

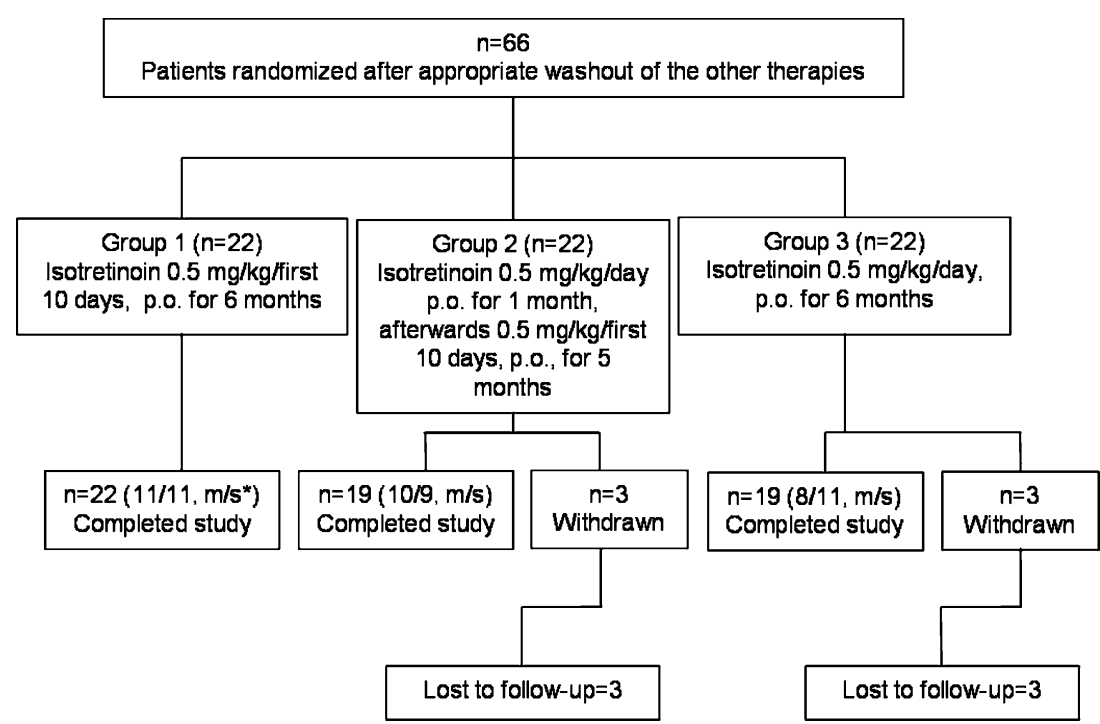


ceptive counselling was done during the treatment and the first 3 months of the follow-up period.

A repeated measure of ANOVA was used to test the acne grading scores and side-effect changes between the study groups. Statistical analysis of the difference within and between the study groups was carried out using the Mann-Whitney $U$-test and Chi-squared with Fisher's exact test at the end of the 6-month treatment and 12-month follow-up period.

\section{Results}

The study was completed on 22 patients in group 1 (19 female, 3 male; mean age \pm SD $22.7 \pm 5.5$ years), 19 patients in group 2 (9 female, 10 male; mean age \pm SD $19.9 \pm 4.9$ years) and 19 patients in group 3 (16 female, 3 male; mean age \pm SD $19.9 \pm 7.3$ years). Six patients failed to continue the follow-up (Fig. 1). Clinical data and total dose of isotretinoin in each group are summarized in Table 1. No statistically significant difference was observed according to age $(P=0.242)$ and gender $(P=0.228)$ among the groups.

The main objective of the study was to asses the efficacy and tolerability of the two different intermittent isotretinoin courses, and compare them with the conventional isotretinoin treatment. Our primary results were obtained at the end of the treatment period (6 months). The clinical evaluation for maintenance efficacy of the treatments during the follow-up period was the final results.

The FDA global grade in each group was found to be significantly decreased compared with baseline values at the end of treatment $(P<0.001)$. Differences were also significant in each group at the end of follow-up period $(P<0.001)$. There were no statistically significant differences between the three treatment groups according to the repeated measure of ANOVA $(P=0.554$; Fig. 2$)$.

However, when patients were evaluated according to the FDA global grade, there was a statistically significant difference between groups 1 and 3 at the end of the 12-month follow-up period $(P=0.002)$. The difference between groups 2 and 3 was also close to the statistical significance $(P=0.053)$. In addition, only in group 1,3 patients $(14 \%)$ had

Table 1 Clinical data and total dose of isotretinoin

\begin{tabular}{llll}
\hline & $\begin{array}{l}\text { Group 1 } \\
(n=22)\end{array}$ & $\begin{array}{l}\text { Group 2 } \\
(n=19)\end{array}$ & $\begin{array}{l}\text { Group 3 } \\
(n=19)\end{array}$ \\
\hline $\begin{array}{l}\text { Age (year) } \\
\text { Sex }\end{array}$ & $22.73 \pm 5.5$ & $19.95 \pm 7.3$ & $19.95 \pm 4.8$ \\
Female $(n)(\%)$ & $14(\% 63.6)$ & $9(\% 47.4)$ & $14(\% 73.7)$ \\
Male $(n)(\%)$ & $8(\% 36.4)$ & $10(\% 52.6)$ & $5(\% 26.3)$ \\
$\begin{array}{c}\text { Mean total dose } \\
(\mathrm{mg} / \mathrm{kg})\end{array}$ & $24.95 \pm 14.7$ & $48.84 \pm 10.6$ & $101.37 \pm 19.2$ \\
\hline
\end{tabular}

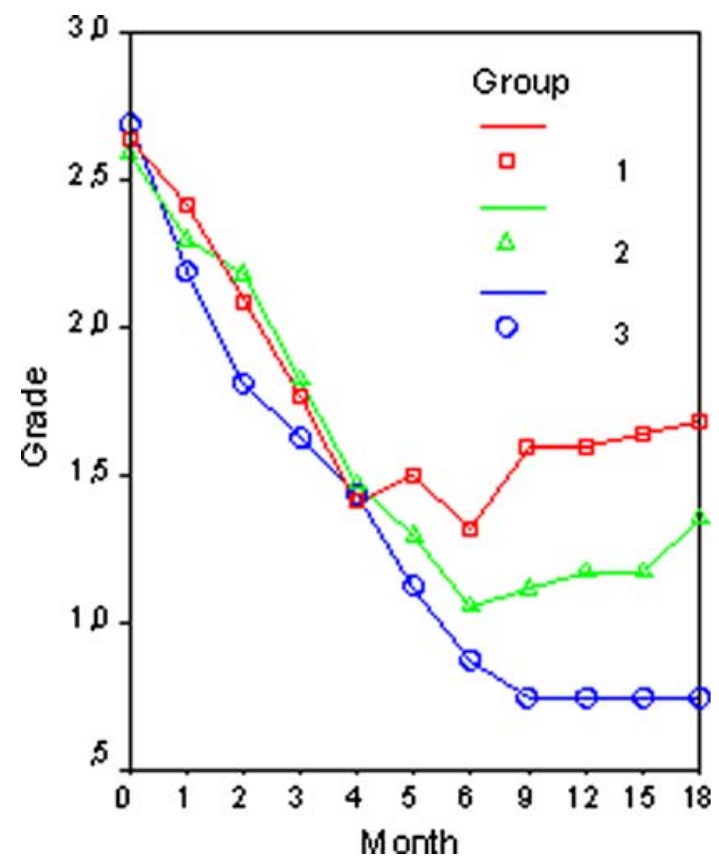

Fig. 2 The repeated measures of FDA global grades of two different intermittent isotretinoin and classical dosage. The graph reflects all included patients. Differences in acne scores were not statistically significant between the groups (0-18 months) according to the repeated measure of ANOVA $(P=0.554)$

relapsed (emergence of pre-treatment acne scores) at the end of the follow-up period; 2 had global grade 3 and 1 had global grade 2 .

When patients were divided into two groups, as moderate and severe, there were no statistically significant differences among the treatment protocols in patients with moderate acne at the end of the follow-up period (group 1 vs. $2, P=0.5$; group 2 vs. $3 ; P=0.15$; group 2 vs. 3 , $P=0.53$ ). However, there was a statistically significant difference between groups 1 and 3 to the response of the treatment at the end of the follow-up period in severe acne patients $(P=0.013)$. The difference between groups 2 and 3 was not statistically significant $(P=0.083$; Fig. 3 ).

As presented in Table 2, the most common side effects were dry, chapped lips (73\% in group 1, 95\% in group 2, $100 \%$ in group 3) and dry skin (59\% in group 1, 65\% in group 2, 78\% in group 3) in all groups. Epistaxis (11\%) and fatigue $(5 \%)$ were reported only in group 3 . Hair loss, bone/ joint aches and pains, muscular cramps or pains were not seen in any group. The frequency of all side effects was higher in group 3 . The dryness in the mouth was significantly higher in group 3 compared with the intermittent groups $(P=0.001)$. The frequency of dry, chapped lips was significantly higher in groups 2 and 3 compared with group $1(P=0.015)$. The dryness of other mucosal tissues $(P=0.044)$ and a rash or facial redness $(P=0.007)$ were significantly higher in group 3 compared with group 1 . 

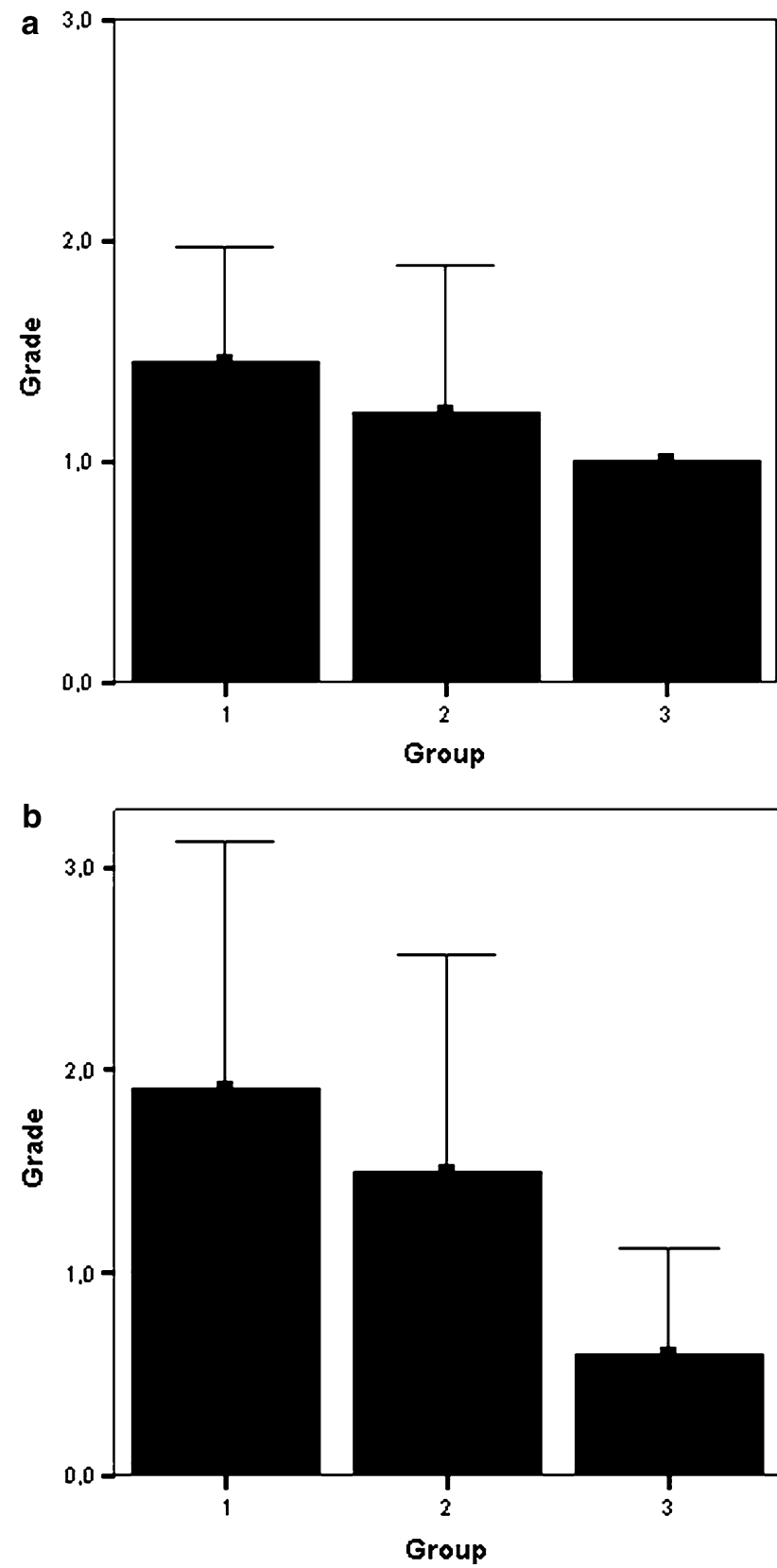

Fig. 3 Differences in FDA global grade for patients with moderate acne $(n=31)$ a and severe acne $(n=29) \mathbf{b}$ at the end of follow-up period. Differences between groups 1 and 3 in severe acne patients was significant $(P=0.013)$

On the basis of data presented in Table 3, the severity scale of all the side effects recorded by physicians, were found to be mild in intermittent groups. Although, the only severity of dry, chapped lips was moderate (Fig. 4), other side effects were mild in group 3 . The differences in dryness in the mouth $(P=0.001)$, dry, chapped lips $(P<0.001)$, dryness of other mucosal tissues $(P=0.026)$, a rash or facial redness $(P=0.004)$, were statistically significant between intermittent groups and the classical dosage group. The differences in pruritus $(P=0.064)$ and the peeling of fingertip skin $(P=0.056)$ were also close to the statistical significance.

Average VAS scores of side effects (except for dry or irritated eyes and excessive thirst) were significantly higher in group 3 compared with intermittent groups (Table 4). Repeated measures of VAS for rash or facial redness presented in Fig. 5. VAS scores, in drug free intervals were milder than on treatment days.

There were no significant abnormalities between groups in terms of liver function tests and fasting lipid values.

\section{Discussion}

In our study, both intermittent isotretinoin treatment courses were found to be as effective as classical dosage during the treatment period with a low incidence and severity of side effects. We did not obtain any significant difference among the treatment protocols in patients with moderate acne. Goulden et al. [5] treated 80 patients with chronic mild and moderate acne, resistant to conventional treatments with isotretinoin $(0.5 \mathrm{mg} / \mathrm{kg} / \mathrm{day})$, for 1 week in every 4 weeks for a total period of 6 months. They reported significant reductions in acne scores. The treatment was well tolerated with mild cheilitis noted as the only side effect. Twelve months following treatment, acne scores remained significantly improved compared with pre-treatment values. In another open and uncontrolled study, Kaymak et al. [6] also used the same intermittent isotretinoin protocol at a dosage of $0.5-0.75 \mathrm{mg} / \mathrm{kg} /$ day in 60 patients with mild and moderate acne localized to the face. This study did not have a follow-up period, and promising results were also reported at the end of the treatment. Mild side effects were noted; however, their prevalence was higher than the study of Goulden et al. [5] The difference was explained by the higher dosages of isotretinoin used in the study of Kaymak et al. In addition to previous intermittent studies, we also included severe cases as well as moderate acne patients. Due to this, we modified the previous protocols, and increased the number of treatment days in each month. Our results, in general, were consistent with the previous two intermittent protocols, and may confirm the effectiveness of this schedule. Additionally, we showed that there was not a significant difference between intermittents and classical dosage treatments at the end of the 6-month treatment period.

When we evaluated the effectiveness of treatment protocols at the end of follow-up period, the decrease in acne scores was statistically significant in all groups. When we investigated the differences among the groups, there was a statistically significant difference in the intermittent group 1 compared with the classical dosage group (group 3). On the other hand, the difference between treatment groups 2 and 3 
Table 2 Frequency of side effects recorded by physicians

a Between group 1-2 and group 3

b Between group 1 and group 2-3

${ }^{c}$ Between group 1 and group 3

Table 3 Average scores for side effects recorded by physicians
Side effects were evaluated on a 4 -point scale as none (0), mild (1), moderate (2) or severe (3)

\begin{tabular}{lcccc}
\hline Side effects & Group $1 n(\%)$ & Group 2n(\%) & Group 3n(\%) & $P$ \\
\hline Mucocutaneous & & & & \\
Dryness in mouth & $2(9)$ & $1(5)$ & $9(47)$ & $0.001^{\mathrm{a}}$ \\
Dry, chapped lips & $16(73)$ & $18(95)$ & $19(100)$ & $0.015^{\mathrm{b}}$ \\
Dry skin & $13(59)$ & $13(65)$ & $14(78)$ & 0.451 \\
Dry or irritated eyes & $2(9)$ & $1(5)$ & $3(16)$ & 0.548 \\
Dryness of other mucosal tissues & $0(0)$ & $1(5)$ & $4(21)$ & $0.044^{\mathrm{c}}$ \\
Pruritus & $3(14)$ & $3(16)$ & $6(32)$ & 0.307 \\
Rash or facial redness & $1(5)$ & $6(32)$ & $9(47)$ & 0.007 \\
Epistaxis & $0(0)$ & $0(0)$ & $2(11)$ & 0.107 \\
Peeling of fingertip skin & $0(0)$ & $2(11)$ & $4(21)$ & 0.081 \\
Other & & & & \\
Hair loss & $0(0)$ & $0(0)$ & $0(0)$ & - \\
Bone/joint aches and pains & $0(0)$ & $0(0)$ & $0(0)$ & - \\
Muscular cramps or pains & $0(0)$ & $0(0)$ & $0(0)$ & - \\
Fatigue & $0(0)$ & $0(0)$ & $1(5)$ & 0.334 \\
Excessive thirst & $2(9)$ & $4(21)$ & $3(16)$ & 0.561 \\
\hline
\end{tabular}

\begin{tabular}{llllr}
\hline Side effects & Group 1 & Group 2 & Group 3 & $P$ \\
\hline Mucocutaneous & & & & \\
Dryness in mouth & 0.09 & 0.05 & 0.47 & 0.001 \\
Dry, chapped lips & 0.71 & 1.16 & 1.95 & $<0.001$ \\
Dry skin & 0.59 & 0.67 & 0.76 & 0.461 \\
Dry or irritated eyes & 0.09 & 0.05 & 0.16 & 0.275 \\
Dryness of other mucosal tissues & 0.00 & 0.05 & 0.21 & 0.026 \\
Pruritus & 0.14 & 0.16 & 0.37 & 0.064 \\
Rash or facial redness & 0.05 & 0.32 & 0.47 & 0.004 \\
Epistaxis & 0.00 & 0.00 & 0.11 & 0.361 \\
Peeling of fingertip skin & 0.00 & 0.11 & 0.32 & 0.056 \\
Other & & & & 0.422 \\
Fatigue & 0.00 & 0.00 & 0.05 & 0.623 \\
Excessive thirst & 0.14 & 0.26 & 0.16 & \\
\hline
\end{tabular}

was also close to the statistical significance. When patients were divided into two groups, as moderate and severe, according to the severity of the disease, there was not a significant difference among the treatment protocols in moderate acne patients at the end of the follow-up period. However, there was a statistically significant difference between groups 1 and 3, for response of the treatments in severe acne patients. In the study of Goulden et al. [5], acne grades remained significantly improved compared with pretreatment values at the end of the 12-month follow-up period. However, $39 \%$ of the patients were reported as relapsed. In our study, only $14 \%$ of patients from the intermittent group 1 reached the pretreatment levels at the end of the follow-up period. Another attribute of our patients in the intermittent groups that differ from the study of Goulden et al. $(21 \mathrm{mg} / \mathrm{kg})$ is that the patients in our study were given a higher cumulative dosage during the treatment ( $25 \mathrm{mg} / \mathrm{kg}$ in group 1 and $49 \mathrm{mg} / \mathrm{kg}$ in group 2). This may explain the lower relapse rates, and again stress the importance of the cumulative dosages of isotretionin to prevent relapses.

In our study, the frequency and severity of isotretioninrelated side effects were found to be lower in group 1 and 2 compared with group 3 . When side effects were evaluated according to treatment protocols, the frequency of side effects in respect to dryness in mucosal tissues, dry and chapped lips and rash/facial redness were significantly lower in both intermittent groups compared to the patients receiving classical 


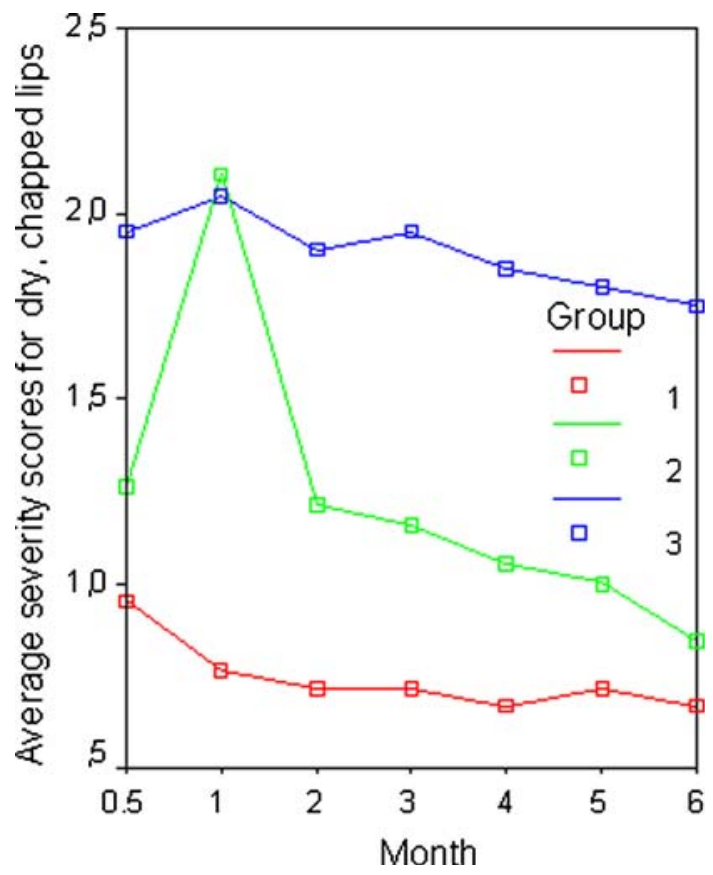

Fig. 4 Repeated measures of average severity scores for dry, chapped lips $(P<0.001)$

dosage. Moreover, the severity scale of side effects recorded by physicians and average VAS scores of side effects, were also found to be milder in intermittent groups. In intermittent groups, we observed a significant decrease in the severity of side effects, especially in drug free intervals, which may explain the increased compliance of these patients to the treatment. Beside neglectable side effects, the other important issue is that the intermittent protocols have lower cost than standard doses. Therefore, lower side effects and treatment cost could encourage patients to use isotretinoin.

Previous studies and our intermittent treatment protocols clearly show the effectiveness of these regimens, especially

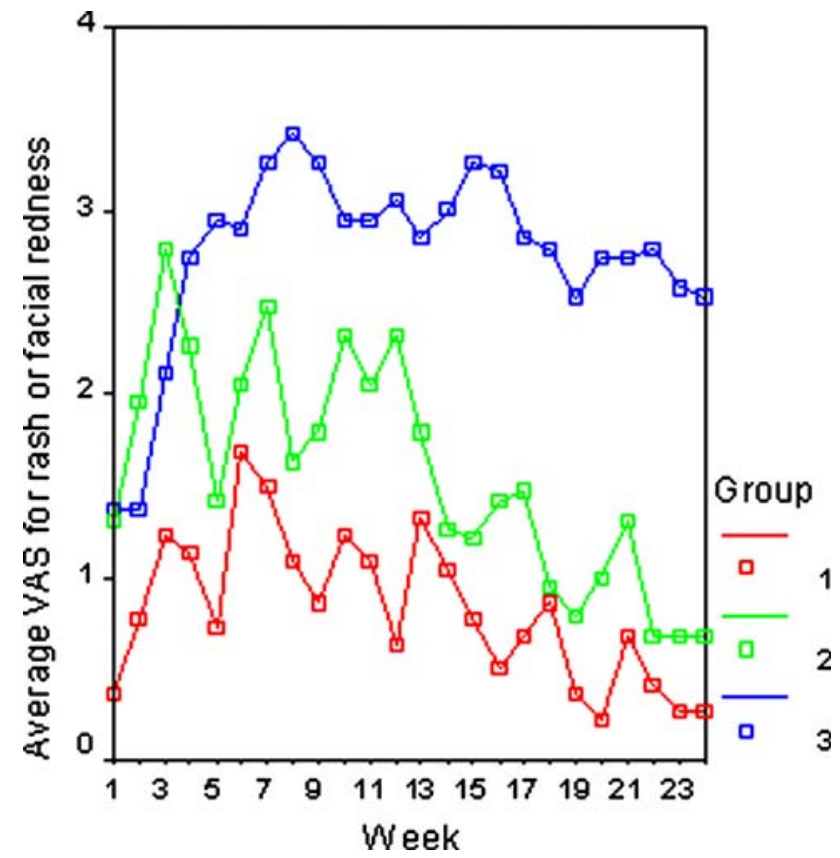

Fig. 5 Repeated measures of VAS for rash or facial redness. Differences in VAS were statistically significant between group 3 and intermittents groups $(P=0.001)$

in mild to moderate acne patients, with a reduced risk of side effects and increased patients compliance. In the light of our results, we can recommend both intermittent isotretionin protocols for those patients with moderate acne, unresponsive to the antibiotic treatment, or who have a tendency to the development of scars. Our study also showed that intermittent treatment protocols were effective in decreasing acne scores in severe acne patients during the treatment. However, we observed an increased rate of recurrences in group 1 during the follow-up period. Therefore, in severe cases, we only suggest intermittent isotretinoin treatment protocol 2 for those patients who do not
Table 4 Average visual analogue scale scores for side effects

\begin{tabular}{llllr}
\hline Side effects & Group 1 & Group 2 & Group 3 & $P$ \\
\hline Mucocutaneous & & & & 0.039 \\
Dryness in mouth & 0.17 & 0.37 & 0.59 & $<0.001$ \\
Dry, chapped lips & 2.09 & 3.16 & 4.63 & 0.011 \\
Dry skin & 1.23 & 1.68 & 2.32 & 0.084 \\
Dry or irritated eyes & 0.05 & 0.58 & 0.53 & 0.015 \\
Dryness of other mucosal tissues & 0.05 & 0.00 & 0.21 & $<0.001$ \\
Pruritus & 0.27 & 0.58 & 1.79 & 0.001 \\
Rash or facial redness & 0.82 & 1.58 & 2.79 & 0.005 \\
Epistaxis & 0.00 & 0.00 & 0.16 & 0.010 \\
Peeling of fingertip skin & 0.05 & 0.16 & 0.53 & 0.002 \\
Other & & & & 0.206 \\
Fatigue & 0.00 & 0.00 & 0.58 & 0.32 \\
Excessive thirst & 0.00 & 0.05 & &
\end{tabular}


tolerate the classical dosage. In patients who have a tendency to increase in acne scores during the follow-up, a new intermittent treatment course, or microdose isotretinoin can be started to reach the sufficient cumulative dosage. In the literature, microdose isotretinoin (20-40 mg/weekly equal to $0.04-0.11 \mathrm{mg} / \mathrm{kg} /$ day) had been tried to prevent the relapse in adult patients suffering from relapse immediately after standard isotretinoin protocol $[1,9]$.

Our study also had some limitations. Because of the study design, there were different investigators, who might have caused some differences in the evaluation of the patients. Furthermore, the follow-up period (12 months) was relatively short.

There is no doubt, that taking a drug at much shorter courses, and lower total doses represents a major therapeutic advance, increases patient compliance, and reduces the risk of side effects. Although oral isotretinoin is the most effective choice for severe acne, its use is associated with many side effects. In this respect, it is needless to say, that intermittent use of isotretinoin may have many advantageous. In our study, treatment of acne with two different intermittent courses was found to be effective, especially in moderate acne with a low incidence and severity of side effects. When the recurrence rates of intermittent courses was evaluated, only intermittent treatment course 2 can be recommended in those severe cases not tolerating the classical dosage. Our results may also indicate that intermittent dose schedule and treatment duration require further evaluation in severe acne, since we have observed a higher rate of relapse compared with classical dosage.

\section{References}

1. Amichai B (2003) Long-term minidoses of isotretinoin in the treatment of relapsing acne. J Dermatol 30:572

2. Amichai B, Shemer A, Grunwald MH (2006) Low-dose isotretinoin in the treatment of acne vulgaris. J Am Acad Dermatol 54:644646

3. Cunliffe WJ, Meynadier J, Alirezai M et al (2003) Is combined oral and topical therapy better than oral therapy alone in patients with moderate to moderately severe acne vulgaris? A comparison of the efficacy and safety of lymecycline plus adapalene gel $0.1 \%$, versus lymecycline plus gel vehicle. J Am Acad Dermatol 49:218226

4. Gollnick HP, Graupe K, Zaumseil RP (2001) Comparison of combined azelaic acid cream plus oral minocycline with oral isotretinoin in severe acne. Eur J Dermatol 11:538-544

5. Goulden V, Clark SM, Mcgeown C, Cunliffe WJ (1997) Treatment of acne with intermittent isotretinoin. Br J Dermatol 137:106-108

6. Kaymak Y, İlter N (2006) The effectiveness of intermittent isotretinoin treatment in mild or moderate acne. J Eur Acad Dermatol Venereol 20:1256-1260

7. Layton AM, Dreno B, Gollnick HP, Zouboulis CC (2006) A review of the European Directive for prescribing systemic isotretinoin for acne vulgaris. J Eur Acad Dermatol Venereol 20:773-776

8. Mandekou-Lefaki I, Delli F, Teknetzis A, Euthimiadou R, Karakatsanis G (2003) Low-dose schema of isotretinoin in acne vulgaris. Int J Clin Pharmacol Res 23:41-46

9. Palmer RA, Sidhu S, Goodwin PG (2000) 'Microdose' isotretinoin. Br J Dermatol 143:205-206

10. Seukeran DC, Cunliffe WJ (1998) Acne vulgaris in the elderly; the response to low-dose isotretinoin. Br J Dermatol 139:99-101

11. Struss JS, Leyden JJ, Lucky AW et al (2001) Safety of a new micronized formulation of isotretinoin in patients with severe recalcitrant nodular acne: A randomized trial comparing micronized isotretinoin with standard isotretinoin. J Am Acad Dermatol 45:196-207 